\title{
Crystal structures and biochemical studies of human lysophosphatidic acid phosphatase type 6
}

\author{
Jun $\mathrm{Li}^{1,2}$, Yu Dong ${ }^{1,2}$, Xingru Lü ${ }^{1}$, Lu Wang ${ }^{1,2}$, Wei Peng ${ }^{1}$, Xuejun C. Zhang ${ }^{1}$, Zihe Rao ${ }^{1,3,4} \bowtie$ \\ ${ }^{1}$ National Laboratory of Biomacromolecules, Institute of Biophysics, Chinese Academy of Sciences, Beijing 100101, China \\ ${ }^{2}$ University of Chinese Academy of Sciences, Beijing 100049, China \\ ${ }^{3}$ Structure Biology Laboratory, Tsinghua University, Beijing 100084, China \\ ${ }^{4}$ Tianjin Key Laboratory of Protein Science, College of Life Sciences, Nankai University, Tianjin 300071, China \\ $\square$ Correspondence: raozh@xtal.tsinghua.edu.cn \\ Received April 18, 2013 Accepted May 28, 2013
}

\begin{abstract}
Lysophosphatidic acid (LPA) is an important bioactive phospholipid involved in cell signaling through Gprotein-coupled receptors pathways. It is also involved in balancing the lipid composition inside the cell, and modulates the function of lipid rafts as an intermediate in phospholipid metabolism. Because of its involvement in these important processes, LPA degradation needs to be regulated as precisely as its production. Lysophosphatidic acid phosphatase type 6 (ACP6) is an LPA-specific acid phosphatase that hydrolyzes LPA to monoacylglycerol (MAG) and phosphate. Here, we report three crystal structures of human ACP6 in complex with malonate, L$(+)$-tartrate and tris, respectively. Our analyses revealed that ACP6 possesses a highly conserved Rossmann-foldlike body domain as well as a less conserved cap domain. The vast hydrophobic substrate-binding pocket, which is located between those two domains, is suitable for accommodating LPA, and its shape is different from that of other histidine acid phosphatases, a fact that is consistent with the observed difference in substrate preferences. Our analysis of the binding of three molecules in the active site reveals the involvement of six conserved and crucial residues in binding of the LPA phosphate group and its catalysis. The structure also indicates a water-supplying channel for substrate hydrolysis. Our structural data are consistent with the fact that the enzyme is active as a monomer. In combination with additional mutagenesis and enzyme activity studies, our structural data provide important insights into substrate recognition and the mechanism for catalytic activity of ACP6.
\end{abstract}

KEYWORDS lysophosphatidic acid, histidine acid phosphatase, crystal structure

\section{INTRODUCTION}

Lysophosphatidic acid (LPA) is the simplest bioactive phospholipid in nature. It consists of a single fatty acyl chain, a glycerol backbone and a free phosphate group. Due to the length of the acyl chain and varying degrees of unsaturation, various structurally diverse forms of LPA exist. LPA is now widely accepted as an important extracellular signal molecule that elicits growthfactor-like activation of G-protein-coupled receptors (GPCRs) (Moolenaar, 1994). LPA has been shown previously to induce a variety of biological functions, including cell growth, neurite retraction, chemotaxis of fibroblasts, membrane depolarization in quiescent fibroblasts (Moolenaar et al., 1992). As an inducer of cell proliferation, migration and survival, LPA also contributes to cancer initiation and progression as well as to metastasis (Mills and Moolenaar, 2003). Recently, the discovery of new LPA receptor types in skeletal cells supports its newly identified role in skeletal biology (Blackburn and Mansell, 2012).

More fundamentally, LPA exists as an intermediate lipid in phospholipid metabolism inside the cell, and plays an important role in modulating the structure and fluidity of lipid rafts. LPA synthesis occurs through a number of pathways. For instance, LPA can be derived from phosphorylation of monoacylglycerol (MAG) by the MAG kinase, or from hydrolysis of phosphatidic acid (PA) by phospholipase A1 or A2 (Kanoh et al., 1986; Thomson and Clark, 1995; Gaits et al., 1997). The acylation of glycerol-3-phosphate (G3P) by G3P acyltransferase is also involved in LPA production (Bell and Coleman, 1980). Another pathway starts from dihydroxyacetone phosphate, which is 
catalyzed by dihydroxyacetone phosphate acyltransferase (Webber and Hajra, 1992), and then the NADPH-dependent acyl-dihydroxyacetone phosphate reductase (Athenstaedt et al., 1999). Furthermore, it is now clear that the biological activity of autotoxin (ATX) is also attributable to the production of LPA from lysophosphatidylcholine (LPC) (Tokumura et al., 2002; Umezu-Goto et al., 2002). In contrast, the degradation of LPA is important for termination of signaling, and for the finetuning of the function of lipid rafts. There are three known LPAmetabolizing pathways. The first is by way of acylation to PA by LPA acyltransferase; the other two through hydrolysis by either LPA phospholipase A (Thompson and Clark, 1994) or LPA phosphatase. Earlier studies of this third pathway have identified an ecto-type LPA phosphatase and a membrane-bound PA phosphatase, both of which are able to hydrolyse LPA as well as other substrates (Kanoh et al., 1992; Xie and Low, 1994; Waggoner et al., 1995; Kai et al., 1996; Waggoner et al., 1996). Lysophosphatidic acid phosphatase type 6 (ACP6) was the first purified and characterized LPA-specific phosphatase (Hiroyama and Takenawa, 1998, 1999). To date, two additional LPA-specific phosphatases have been identified and purified from plants (Shekar et al., 2002; Reddy et al., 2010).

Previous studies have shown that ACP6 is localized in the mitochondria with ubiquitous expression throughout all tissues, and high expression levels in kidney, heart, small intestine, muscle, and liver. Through specifically hydrolyzing LPA, ACP6 regulates mitochondrial lipid biosynthesis, thereby controlling important mitochondrial functions (Hiroyama and Takenawa, 1999). It was also shown that ACP6 might be involved in tumor progression, suggesting that ACP6 could be a good candidate marker for the prognosis of esophageal cancer (Ando et al., 2006). However, the structural basis of its substrate recognition, and the mechanism of catalysis remained unclear. Here, we present for the first time, three crystal structures of human ACP6 in complex with either malonate, $L(+)$-tartrate or tris. Analysis of these structures, in combination with the results from biochemical studies have provided us with a better understanding on these aspects.

\section{RESULTS}

\section{Overview of ACP6 structure}

The structures of ACP6-malonate, ACP6-tris and ACP6-L-(+)tartrate complexes were solved by Se-SAD or MR method (Table 1). The final $R_{\text {work }}$ values were $17.6 \%, 16.9 \%$ and $20.1 \%$, respectively (with $R_{\text {free }}$ values of $22.3 \%, 20.1 \%$ and $24.9 \%$ ). All crystals belong to the $P 22_{1} 2_{1} 2_{1}$ space group, and only one molecule of ACP6 is in the asymmetric unit, with Matthews coefficients of $2.07 \AA^{3} / \mathrm{Da}, 2.66 \AA^{3} / \mathrm{Da}$ and $2.50 \AA^{3} / \mathrm{Da}$, respectively (the corresponding solvent contents were $41 \%$, $54 \%$ and $51 \%$ ) (Matthews, 1968). The three structures were nearly identical by superposition. The r.m.s.d. between ACP6malonate and ACP6-tris was $0.5 \AA$ for 313 residue pairs, while it was $0.3 \AA$ between ACP6-L-(+)-tartrate and ACP6-tris when 337 residue pairs were compared.
The garlic flake-shaped structure of ACP6 has an overall dimension of $71 \AA \times 52 \AA \times 41 \AA$, and can be divided into two domains (Fig. $1 \mathrm{~A}$ and $1 \mathrm{~B}$ ). The Rossmann-fold-like body domain is similar to the classic $\alpha / \beta$ core domain of enzymes belonging to the histidine acid phosphatase superfamily, including a seven-stranded $\beta$-sheet in the middle, two $\alpha$-helices $(\alpha 2, \alpha 3)$ on one side and four helices $(\alpha 9, \alpha 10, \alpha 11, \alpha 12)$ on the opposite. The $\beta$-strands are arranged in parallel with an order of $\beta 2-$ $\beta 4-\beta 3-\beta 5-\beta 1-\beta 6-\beta 7$, except that $\beta 6$ is antiparallel. Compared to the classic $\alpha / \beta$ core domain, $\beta 2$ represents an additional strand, which is inserted between $\beta 1$ and $\beta 3$. Viewed from $\beta 7$ to $\beta 2$, the strands exhibit a decrease in length towards $\beta 2$, with the $\beta$ sheet twisting counterclockwise for about $107^{\circ}$. Most of the helices around the sheet are approximately vertically arranged, except for the inclined $\alpha 10$ and the bent $\mathrm{N}$-terminus of $\alpha 9$. Such three-layered structure makes the body domain a stable core of the enzyme. The $a$-helices-only cap domain covers the body domain, which contains three sections: 1 ) the part of the loop between $\beta 1$ and $\beta 2$ that covers residues positioned from 63 to 81 ; 2) $\alpha 1$ and the following loop; and 3) a helix bundle ( $\alpha 4-\alpha 8$ ) inserted between $\beta 4$ and $\alpha 9$ of the body domain. The interaction between $\alpha 6$ and section 1 locks the bundle of helices in an inclined position, forming a cap on top of the body domain. Helix $\alpha 1$ is also restrained on the other side of section 1 by the interaction of its following loop with section 1 , but it is missing in structures of ACP6-malonate and ACP6-L(+)-tartrate, implying its positional flexibility. The junction of the two domains forms a large substrate-binding pocket as well as a hole in the structure (Fig. 1C). There are two conserved disulfide bridges in the structure. One is formed between Cys208 and Cys416, linking a12 at the C-terminal of the peptide chain and $\alpha 4$ in the middle of the chain. It is noted that in the ACP6-tris structure, the distance between the corresponding $S$ atoms is $3.84 \AA$, which is beyond the limit of disulfide bond formation, possibly caused by reduction during X-ray diffraction. The other bond is between Cys388 and Cys393, locking a five-residue turn outside $\beta 7$ strand. Both disulfide bridges participate in stabilizing local structures. By checking the $B$ factor for every residue in the molecule, we found that the inner residues, especially those around the active site, are less flexible compared to those at the surface. Thus, the whole 45-kDa large architecture strongly supports the structure of the substrate-binding pocket, together with the active site within, which is required for catalysis.

A 3D structure alignment-based similarity search against the PDB database provided by the Dali server showed that the most similar homologous structure of ACP6 is human prostatic acid phosphatase (hPAP), which shares $25 \%$ sequence identity with ACP6, with an r.m.s.d. of $3.7 \AA$ between the 270 residue pairs (LaCount et al., 1998; Jakob et al., 2000; Ortlund et al., 2003). Other significant hits included histidine acid phosphatase from Francisella tularensis (FtHAP), phytase and glucose-1-phosphatse from Escherichia coli, all of which belong to the histidine acid phosphatase superfamily (Lim et al., 2000; Lee et al., 2003; Singh et al., 2009). Our structure provides 
Table 1. Data collection and refinement statistics

\begin{tabular}{|c|c|c|c|}
\hline Parameters & ACP6-malonate (Se-Met) & ACP6-tris (Se-Met) & ACP6-L-(+)-tartrate \\
\hline \multicolumn{4}{|l|}{ Data collection } \\
\hline Space group & $P 2_{1} 2_{1} 2_{1}$ & $P 2_{1} 2_{1} 2_{1}$ & $P 2_{1} 2_{1} 2_{1}$ \\
\hline \multicolumn{4}{|l|}{ Unit-cell parameters } \\
\hline$a, b, c(\AA)$ & $42.6,87.9,90.1$ & $53.0,90.7,95.7$ & $44.3,91.0,105.0$ \\
\hline$\alpha, \beta, \gamma\left({ }^{\circ}\right)$ & $90.0,90.0,90.0$ & $90.0,90.0,90.0$ & $90.0,90.0,90.0$ \\
\hline Wavelength $(\AA)$ & 0.9787 & 0.9791 & 1.0000 \\
\hline Resolution $(\AA)$ & $50.00-2.20(2.28-2.20)^{a}$ & $50.00-2.20(2.28-2.20)$ & $50.00-2.17(2.25-2.17)$ \\
\hline Total observation & 120,658 & 166,427 & 153,954 \\
\hline Unique reflections & $32,627^{b}$ & $44,371^{\mathrm{b}}$ & 23,126 \\
\hline Data completeness (\%) & $99.9(99.6)$ & $99.9(100.0)$ & $99.9(99.3)$ \\
\hline$R_{\text {merge }}^{\mathrm{c}}$ & $0.099(0.480)$ & $0.095(0.457)$ & $0.085(0.457)$ \\
\hline$\| / \delta(l)$ & $15.6(3.9)$ & $16.9(5.5)$ & $19.2(3.7)$ \\
\hline Redundancy & $6.9(5.9)$ & $7.0(7.3)$ & $6.6(5.8)$ \\
\hline \multicolumn{4}{|l|}{ Refinement } \\
\hline Resolution $(\AA)$ & $45.07-2.21$ & $46.39-2.21$ & $45.51-2.17$ \\
\hline Reflections used & 17,186 & 22,918 & 21,832 \\
\hline$R_{\text {work }} / R_{\text {free }}{ }^{d}$ & $0.176 / 0.223$ & $0.169 / 0.201$ & $0.201 / 0.249$ \\
\hline \multicolumn{4}{|l|}{ No. of non-H atoms } \\
\hline Protein & 2,939 & 3,046 & 2,986 \\
\hline Ligand & 7 & 8 & 10 \\
\hline Water & 69 & 136 & 75 \\
\hline \multicolumn{4}{|l|}{ Average $B$ factor $\left(\AA^{2}\right)$} \\
\hline Protein & 41.9 & 39.6 & 52.7 \\
\hline Ligand & 53.2 & 47.7 & 51.3 \\
\hline Water & 41.2 & 41.9 & 46.6 \\
\hline \multicolumn{4}{|l|}{ R.m.s. deviation } \\
\hline Bond lengths $(\AA)$ & 0.006 & 0.006 & 0.009 \\
\hline Bond angles $\left({ }^{\circ}\right)$ & 1.110 & 0.996 & 0.911 \\
\hline \multicolumn{4}{|l|}{ Ramachandran plot (\%) } \\
\hline Favored & 97.4 & 99.5 & 98.3 \\
\hline Allowed & 2.6 & 0.5 & 1.7 \\
\hline Outliers & 0 & 0 & 0 \\
\hline
\end{tabular}

${ }^{a}$ Values in parentheses are for the highest resolution shell.

${ }^{\mathrm{b}}$ The numers of $\mathrm{I}(+)$ and $\mathrm{I}(-)$ reflections are counted separately and summed together.

${ }^{\mathrm{c}} R_{\text {merge }}=\Sigma_{\mathrm{h}} \Sigma_{\mathrm{i}} l_{\mathrm{ih}_{\mathrm{h}}}-<l_{\mathrm{h}}>\mid \Sigma_{\mathrm{h}} \Sigma_{\mathrm{i}}<l_{\mathrm{h}}>$, where $\left\langle l_{\mathrm{h}}>\right.$ is the mean intensity of the observations of $l_{\text {ih }}$ of reflection $\mathrm{h}$.

${ }^{\mathrm{d}} R_{\text {work }}=\Sigma_{\mathrm{h}} \mid F_{\mathrm{o}}-F_{\mathrm{c}} / \Sigma_{\mathrm{h}} F_{\mathrm{o}}$, where $F_{\mathrm{o}}$ and $F_{\mathrm{c}}$ are the observed and calculated structure factor amplitudes of reflection $\mathrm{h}$. $R_{\text {free }}$ is mathematically equivalent to $R_{\text {work }}$, but was measured over $8 \%$ of the data.

further evidence that ACP6 is indeed a member of the histidine acid phosphatase superfamily. Multiple sequence alignment and structure superposition showed that the body domain of ACP6 is conserved, while the cap domain is much more different from homologous structures (Figs. 1D and 2).

\section{ACP6 is active as a monomer}

Unlike the dimeric PAP and FtHAP, ACP6 mainly appeared in its monomeric form in solution when analyzed by gel filtration (Fig. S1). Also, our structural data indicates that there is only one molecule in the asymmetric unit. Performing a PISA analysis (Krissinel and Henrick, 2007), we found that the largest interface area buried by the symmetry-related molecules is $633 \AA^{2}$, with a complex formation significance score of 0.0 , which means the interface is just a result of crystal packing. Thus, no evidence was collected that dimers exist within the 


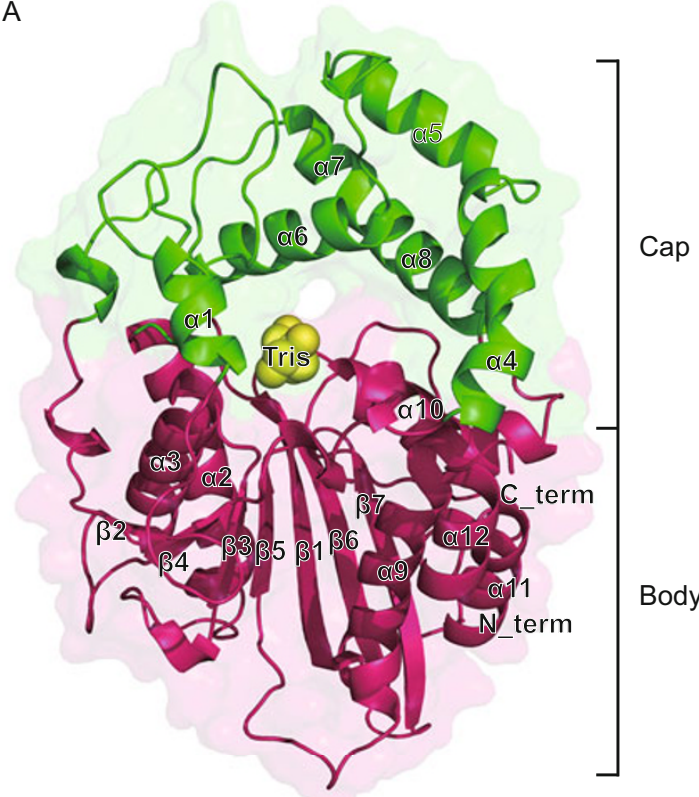

C

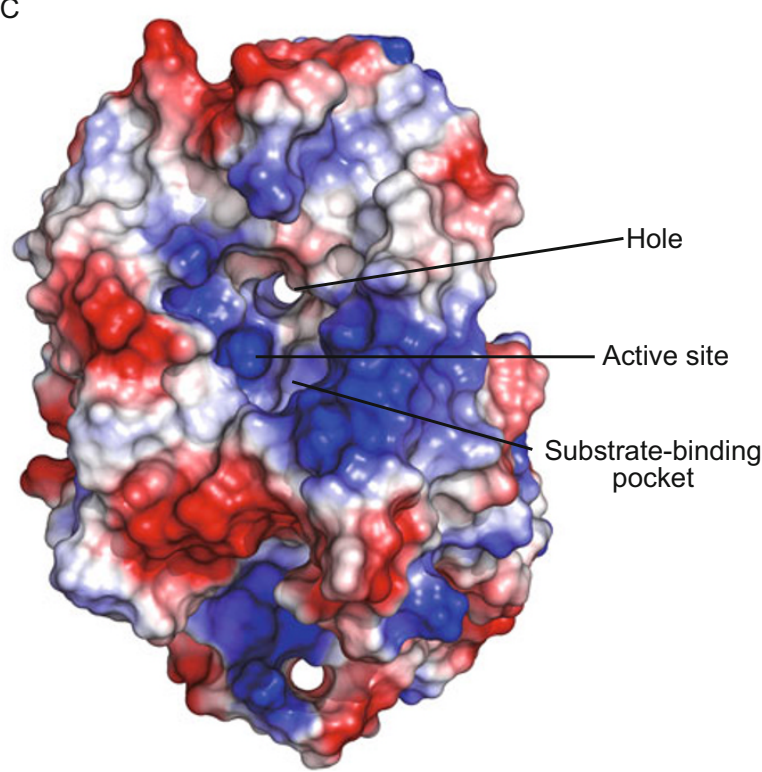

B

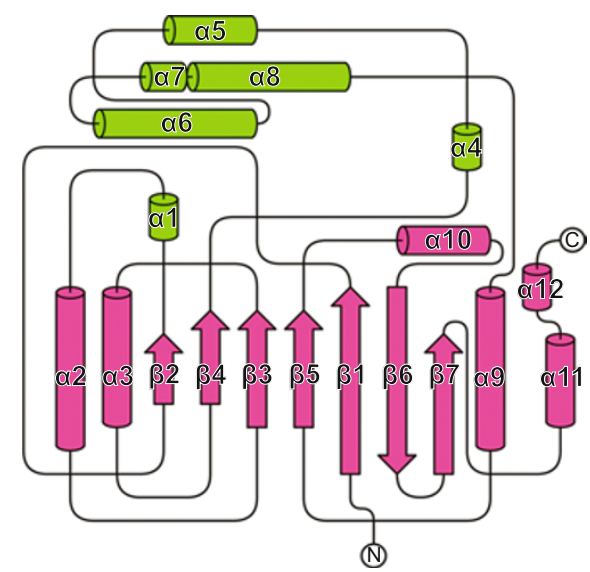

D

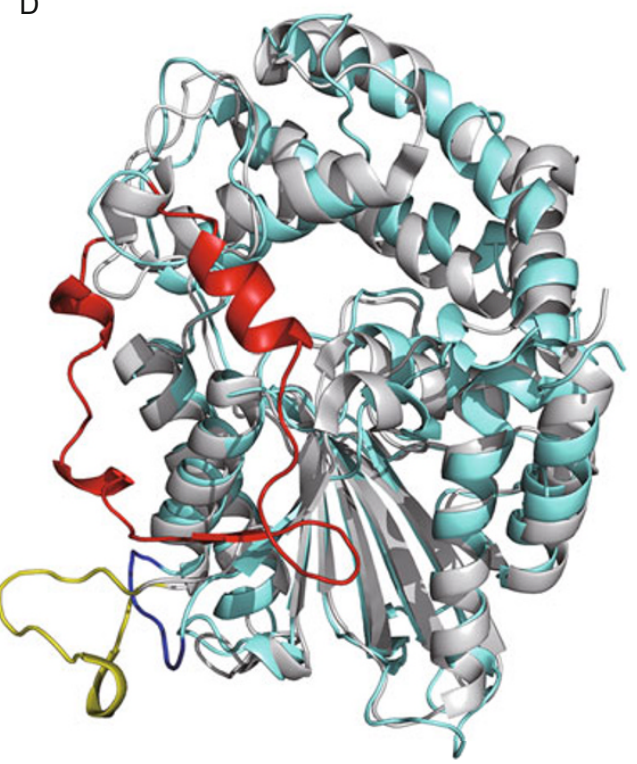

Figure 1. Overall structure of ACP6. (A) Ribbon and surface representations of ACP6. The body domain is shown in pink, and the cap domain is shown in green. The tris ligand is indicated as spheres. (B) Topology diagram of ACP6. The colors of the body domain and the cap domain are as same as (A). (C) Electrostatic potential surface of ACP6. Blue, red and white colors represent positively charged regions, negatively charged regions, and neutral zones, respectively. (D) Structure superposition of ACP6 (cyan) and hPAP (grey). The protruding loop of hPAP is shown in yellow; the shorter loop at the same position in ACP6 is shown in blue. The additional fragment 77-116 of ACP6 is shown in red.

crystal lattices. In the case of dimeric rat prostatic acid phosphatase (rPAP), two residues important for oligomerization, were found to be Trp137 and His143 (Porvari et al., 1994). Trp137 is located in a protruding loop of the rPAP structure, which interacts with another Trp137 in the dimeric subunit through stacking of their aromatic rings (Fig. 3A). In contrast, a short loop between $\alpha 3$ and $\beta 4$ takes place of the protruding loop in ACP6 thus the corresponding residue and interaction are missing (Fig. 3B). Furthermore, His192 in $\beta 4$, which corresponds to His143 of rPAP, is blocked by a turn in the loop between $\beta 2$ and $\alpha 1$, and in addition, Asp107 interacting with His143 in rPAP is replaced by Asn165 in ACP6. Moreover, an additional triangular structure (fragment 77-116) was observed to block the corresponding dimer interface completely. In con- 

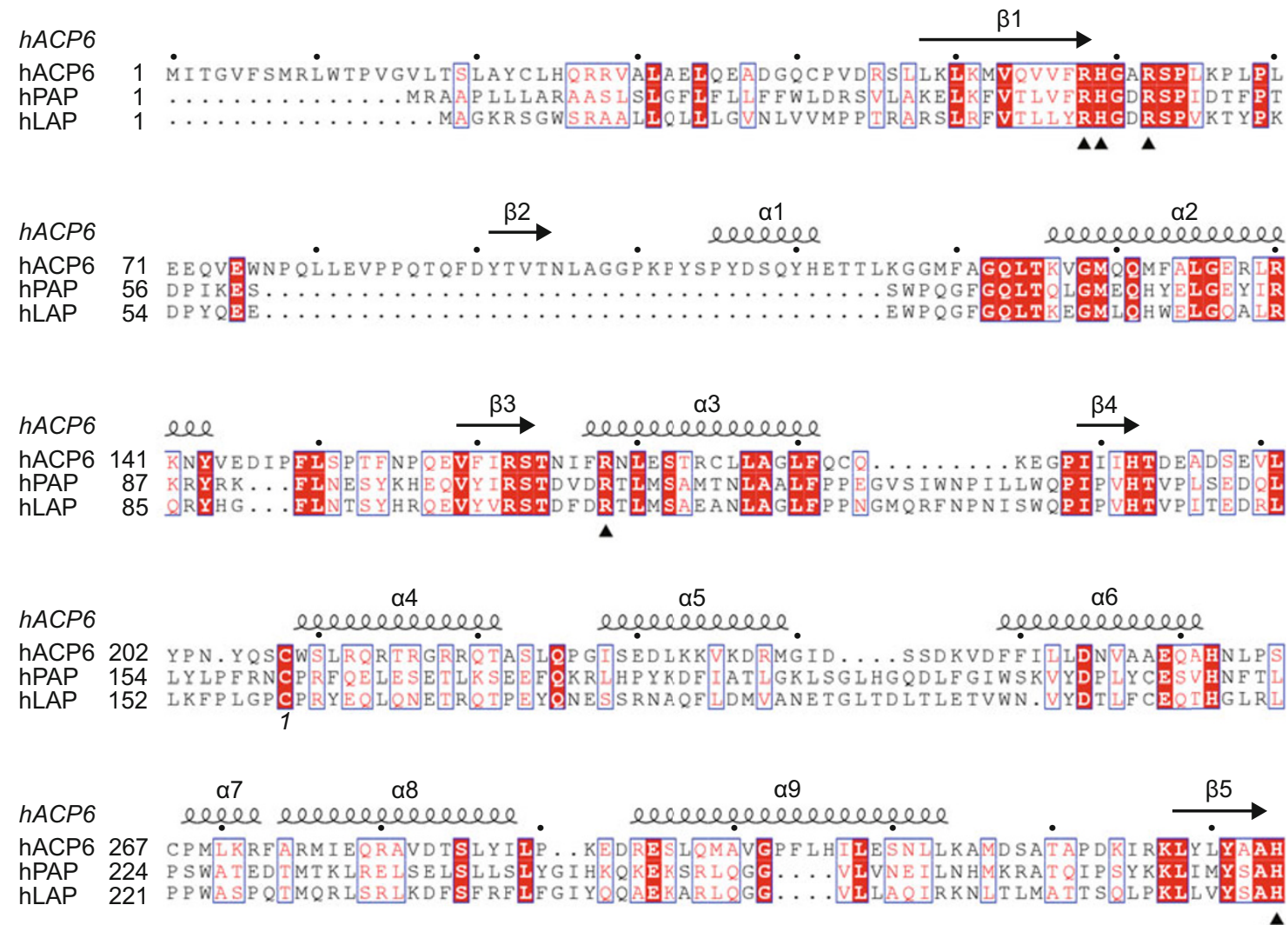
hLAP
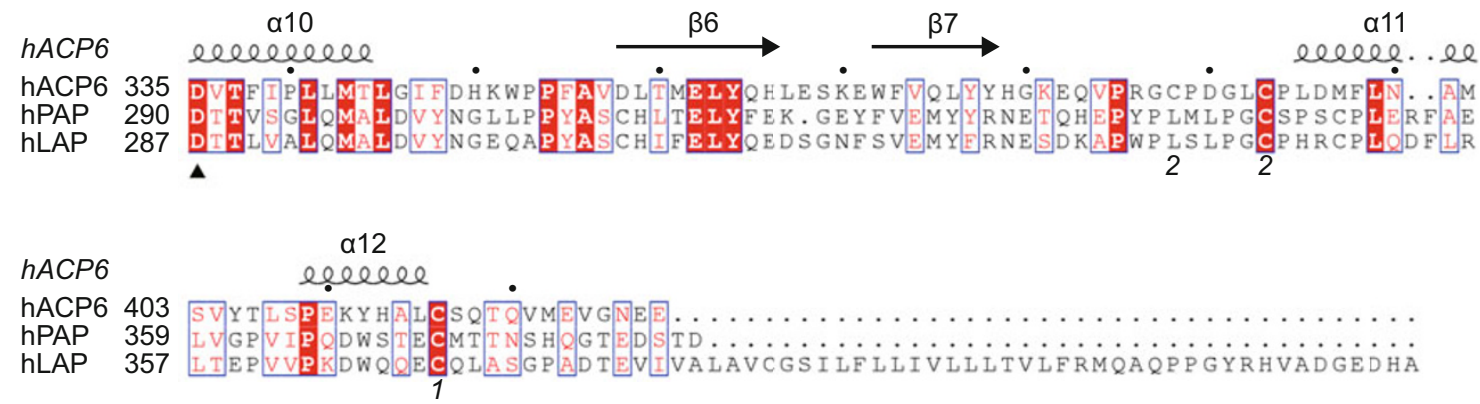

Figure 2. Sequence alignment of hACP6 with hPAP and hLAP. Secondary structures of ACP6 are labeled on top of the aligned sequences. Residues in the active site are marked with $\boldsymbol{\Delta}$. Cysteines forming disulfide bonds are marked with pairs of numbers. hPAP, human prostatic acid phosphatase; hLAP, human lysosomal acid phosphatase.

clusion, ACP6 is not able to dimerize and is likely to be soluble only as a monomer. It is known that dimerization is indispensable for the activity of PAP. Whilst the deletion of the triangular structure will cause inhomogeneous oligomerization and loss of activity (Figs. 4C and S1), the presence of such structure, instead of dimerization, should keep ACP6 active in its monomeric form.

\section{The substrate-binding pocket}

Though similar structures are shared within the histidine acid phosphatase superfamily, their substrate-binding pockets are of different shape, thus functioning in recognizing and hydrolyz- ing different kinds of substrates. For instance, PAP has a small and shallow pocket with two stretching grooves for PNPP, LPA and PA (Fig. 5A). FtHAP has a narrow pocket clamping 3'-AMP (Fig. 5B). In contrast, ACP6 possesses a large pocket that is only suitable for LPA but not for other substrates (Fig. 5C), which was confirmed by our enzyme activity studies (Fig. S2) and by results from a previous report (Hiroyama and Takenawa, 1999). Smaller molecules such as PNPP, 3'-AMP, 5'-AMP are not preferred substrates, because there are no suitable residues near the phosphate group-binding site to stabilize (or clamp) their small alcohol group in ACP6 compared to PAP and FtHAP. On the other hand, there is no space for more than one acyl chain in the pocket, indicating that PA may 

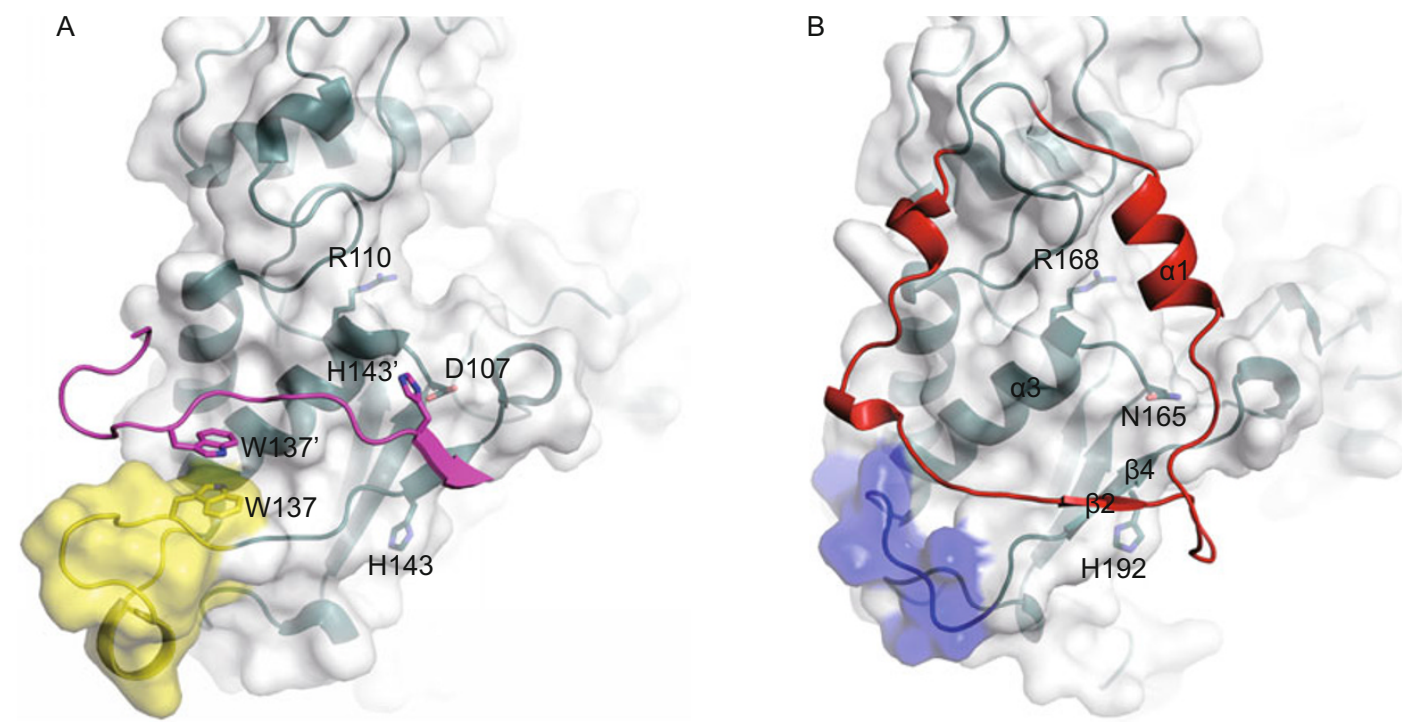

Figure 3. Structural basis of monomeric ACP6 and dimeric rPAP. (A) Dimer interactions of rPAP. (B) The different structure features in ACP6 from the same view as (A). The protruding loop of rPAP is shown in yellow; the shorter loop at the same position in ACP6 is shown in blue. The additional fragment $77-116$ of ACP6 is shown in red. The interacting fragment in the other dimeric subunit of rPAP is labeled in magenta. The side chains of relevant residues are presented as sticks.

not be a preferred substrate either. To inspect how the pocket is formed, we compared the structure of ACP6 with hPAP. The superposition showed that the existence of $\alpha 1$ increases the height of the left-front side wall of the pocket, which deepens the active site within (Fig. 5D). The fragment 216-225 between $\alpha 4$ and a5 in ACP6 blocks one groove stretching from the pocket of hPAP. Compared to hPAP, the $a 6$ of ACP6 has a shorter N-terminus, and the C-terminus of a8 shifts backwards. All these differences contribute to the shape of the right half of the ACP6 pocket. The other groove of hPAP is also blocked in ACP6, namely by the side chains of a U-shaped loop between $\beta 4$ and $\alpha 4$. Furthermore, hydrophobic residues inside the pocket create a hydrophobic environment (Fig. 5D). Thus, based on these structural features, it is possible for ACP6 to readily fit and hold the long acylglyceroyl group of LPA inside its pocket.

\section{The active site}

The active site of ACP6, composed of six conserved residues including Arg58, His59, Arg62, Arg168, His334 and Asp335, presents a positively charged area inside the substrate-binding pocket on the surface presentation of electrostatics (Fig. $5 \mathrm{C}$ ), which is suitable for binding the negatively charged phosphate group of the substrate. Interestingly, in each of our solved structures, there is a small molecule occupying this site. Malonate and L-(+)-tartrate were constituents of the crystallization reservoir, whereas tris was a component of the protein buffer.

In the case of the ACP6-malonate complex, one carboxyl moiety of malonate mainly interacts with the guanidinium group of Arg58 through a pair of nearly parallel salt bonds, while the other carboxyl moiety mainly interacts with Arg168 in identical fashion (Fig. 5E). Other residues also participate in malonate binding, but the interactions are relatively weak, as inferred from the unfavorable bond angles. In the case of the ACP6-L(+)-tartrate complex, the carboxyl group on one end protrudes from the active site due to the larger size of tartrate (Fig. 5F). The other carboxyl group in the center mainly interacts with the guanidinium group of Arg58. However, the two groups are not in the same plane, possibly due to the interaction of the distal hydroxyl and carboxyl groups with His334 and Arg168. In fact, four $\mathrm{O}$ atoms from two hydroxyl groups and the central carboxyl group form the four apexes of a tetrahedron conformation, interacting with surrounding residues and stabilizing the molecule in its position. The tetrahedron conformation here resembles the $P_{i}$ binding manner in FtHAP and hPAP (PDB code: 3IT0 and 1ND6) (Ortlund et al., 2003; Singh et al., 2009). The interaction between ACP6 and L-(+)-tartrate resembles that observed in the structure of FtHAP complexed with L-(+)tartrate (PDB code: 3IT1) (Singh et al., 2009). In the case of the ACP6-tris complex, all three hydroxyl group arms of tris are interacting with surrounding residues through hydrogen bonds. Two of the arms mainly interact with Arg58 in parallel, and the third arm is immobilized by Arg168 and His334 (Fig. 5G). The positively charged amino group points to the bulk solvent of the pocket, and interacts with Asp335, as well as an adjacent water molecule. Interestingly, the four arms of tris also present a tetrahedron conformation that resembles $\mathrm{P}_{\mathrm{i}}$, but is more apparent than that of L-(+)-tartrate. In conclusion, analysis of the binding manners of the three molecules has shown that four residues in the active site (Arg58, Arg62, Arg168 and His334) function together as a positively charged paw, which clamps the phosphate group as well as these small molecules. Furthermore, the proton bound to the $\mathrm{N} \varepsilon$ atom of His59 is donated 
A

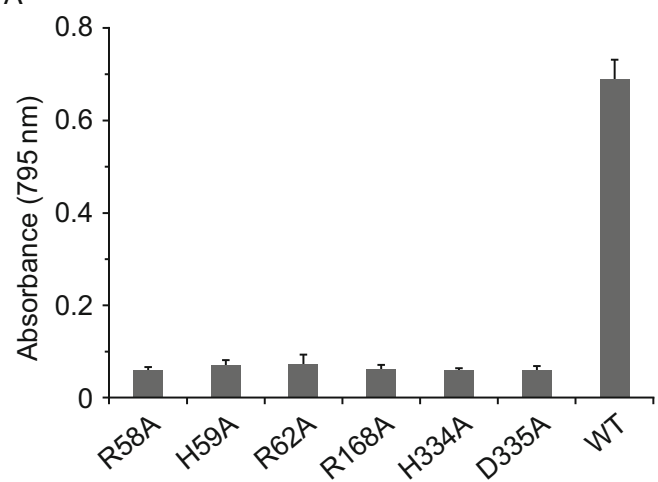

C

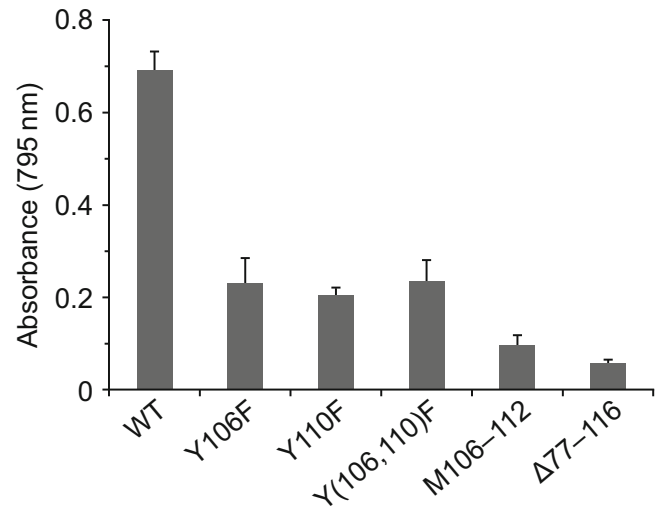

$\mathrm{E}$

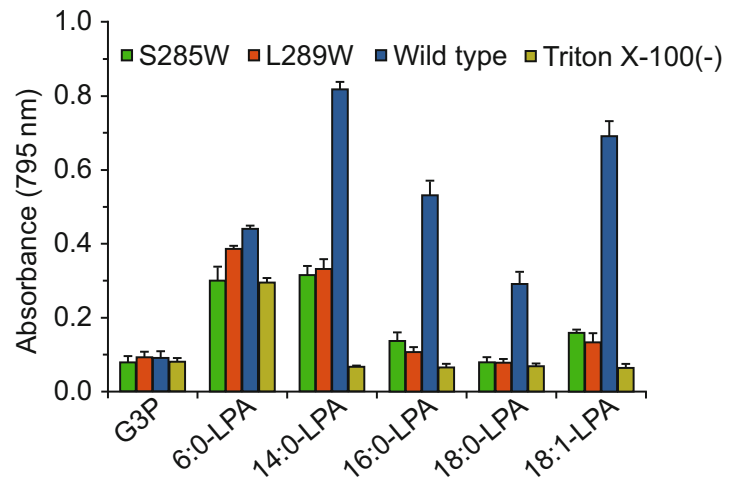

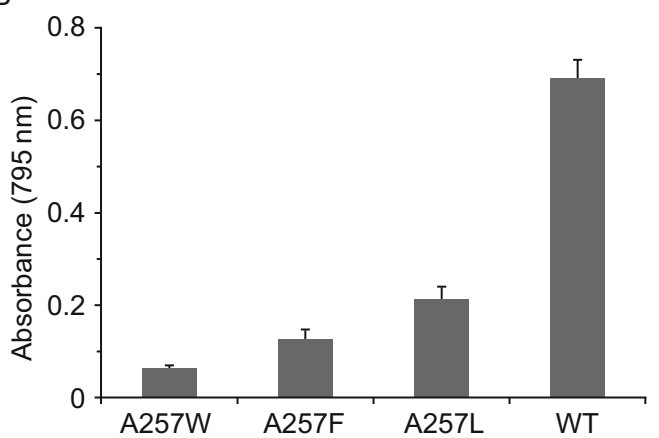
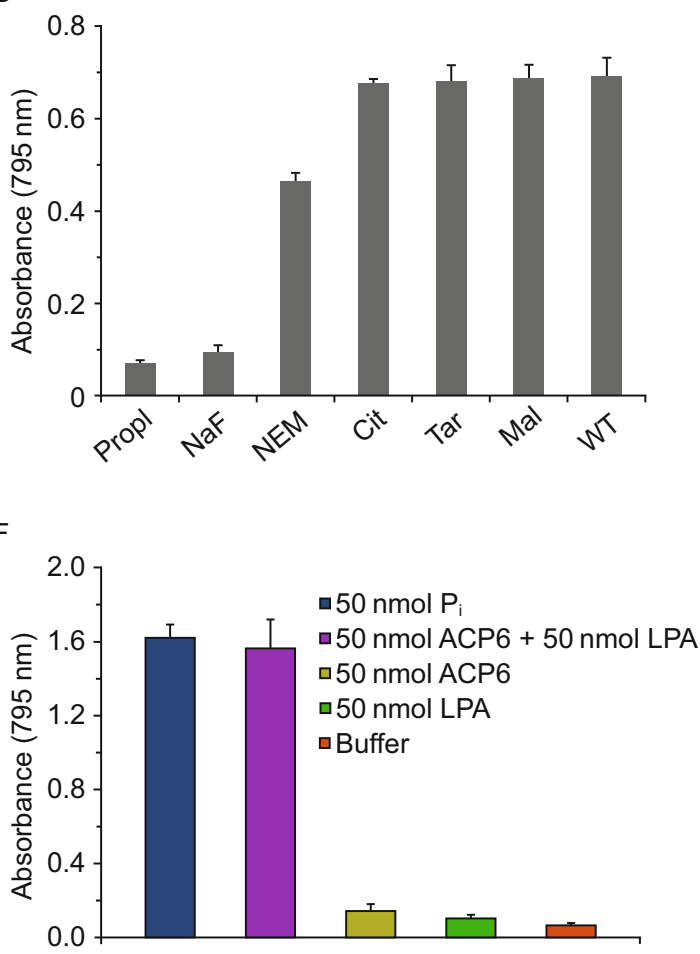

Figure 4. Enzyme activity assay. (A) Enzyme activity of residue mutations in the active site. (B) Enzyme activity of different A257 mutations at the entrance of water-supplying channel. (C) Enzyme activity of mutations in the additional fragment 77-116. (D) Inhibitory effect of different molecules. (E) Enzyme activity on different LPAs plus G3P. (F) Modified enzyme activity assay without Triton X-100. Error bars represent s.d. $(n=3)$. By default, 18:1-LPA is used as standard substrate in enzyme activity assay. WT, wild type; Propl, propranolol; NaF, sodium fluoride; NEM, N-ethylmaleimide; Cit, citrate; Tar, L-(+)-tartrate; Mal, malonate.

to the three surrounding alkaline arginines. This fact, together with its position, turns the $\mathrm{N} \varepsilon$ atom of His59 into the nucleophile of the enzyme. On the other hand, the $\mathrm{N}^{\prime}$ atom of tris is located opposite to the side of the nucleophilic attack and orients towards the bulk solvent of the pocket, which suggests that the leaving alcohol group of the substrate is covalently bound to the phosphate group at the corresponding position of the $\mathrm{N}^{\prime}$ atom. Given that the $\mathrm{pK}_{\mathrm{a}}$ of aspartic acid is 3.9 , Asp335 should be deprotonated at physiological $\mathrm{pH}$ conditions, and therefore should be unable to protonate the leaving alcohol group of the substrate. It is worth noting that Asp335 interacts with a water molecule near the $\mathrm{N}^{\prime}$ atom. This water is considered to be activated by Asp335, to protonate the leaving alcohol group of substrate and then release $\mathrm{P}_{\mathrm{i}}$. Mutation of any of the six conserved residues in the active site to alanine resulted in a complete loss of enzyme activity (Fig. 4A), which is consistent with their contribution in phosphate group binding and hydrolysis, as inferred from structural analysis. 

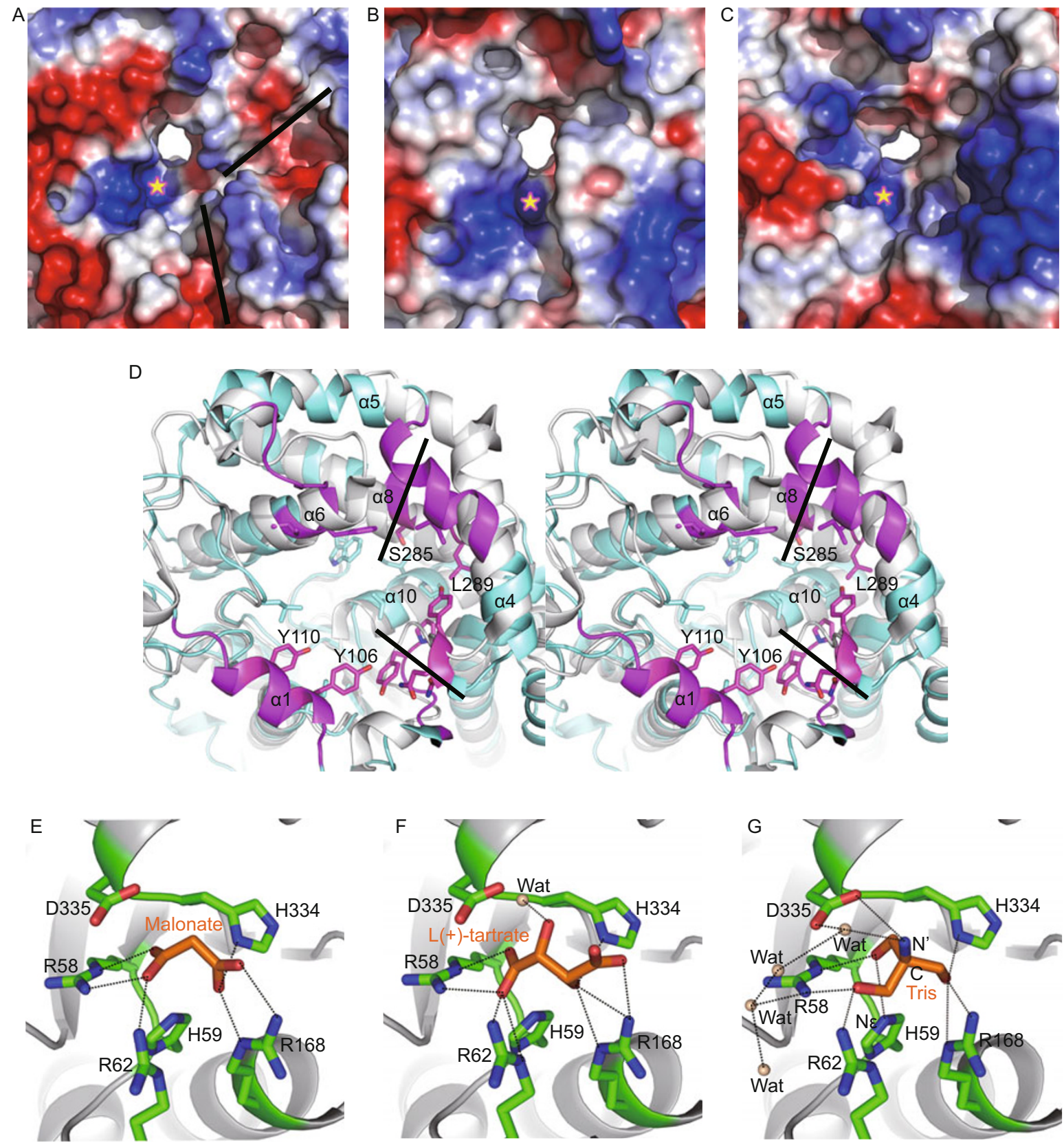

Figure 5. The substrate-binding pocket and the active site of ACP6. Substrate-binding pockets of hPAP (A), FtHAP (B) and ACP6 (C) are compared in the surface presentation of electrostatics. The active site is marked with th, grooves in hPAP are marked with black lines. (D) Stereo view of the substrate-binding pockets of ACP6 (cyan) and hPAP (grey) in superposition. The different structures in the pocket of ACP6 compared with hPAP are colored as magenta. The side chains of hydrophobic residues and other mentioned residues in the pocket are shown in stick representation. The grooves in hPAP are marked with black lines. Malonate $(E), L(+)$-tartrate $(F)$ and tris (G) are present in the active site of ACP6. Ligands and side chains of residues in the active site are shown in stick representation. Water molecules are shown in spheres. Interactions are marked with dashed black lines.

\section{Water-supplying channel}

In the surface representation of ACP6, a channel is located above the active site, connecting the substrate-binding pocket with the bulk solvent on the backside of the molecule
(Fig. 6A). This $7.5 \AA$ long channel, with a diameter of about $7.0 \AA$, is constituted by residues from a6 (Leu253, Asp254, Ala257), a8 (Glu278), the loop between $\alpha 10$ and $\beta 6$ (Trp352, Pro353, Pro354, Phe355), and Arg58 in the active site, which makes the only hydrophilic part inside the channel (Fig. 6B). 
A

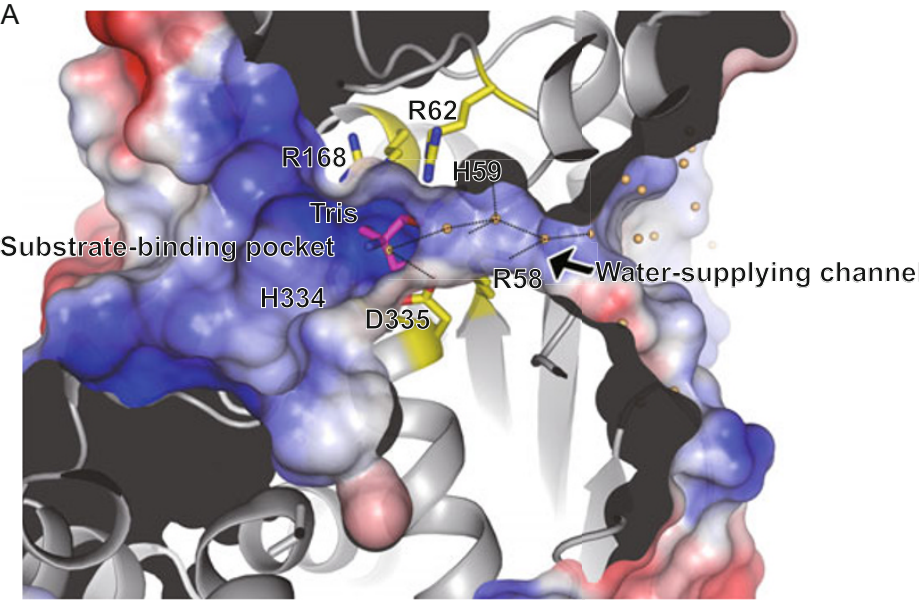

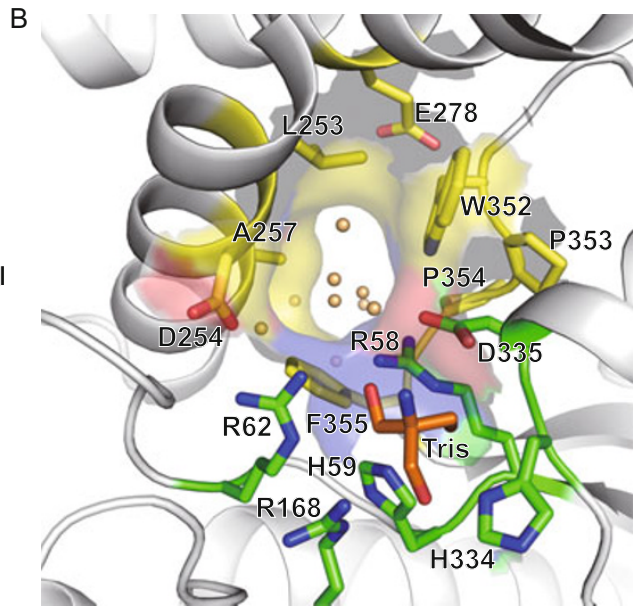

Figure 6. Water-supplying channel. (A) Cutaway view of the channel showing a water molecule chain inside it. ACP6 is present in the diagram of ribbon and surface of electrostatic potential. Interactions are marked with dashed black line. (B) The surface representation of the channel showing its residue composition. Tris and side chains of relevant residues are shown in stick representation. Water molecules are shown in spheres.

In ACP6-tris, six water molecules are observed in this channel. Through hydrogen bond interaction, five interact with each other in tandem and form a water molecule chain. Within the channel, only Arg58 participates in the interaction with two water molecules, and the terminal water molecule of the chain is caught by Asp335. There are also abundant water molecules near the entrance of the channel on the backside surface (Fig. 6A). Taken together, these structural features suggest that the channel is for supplying water from the bulk solvent on the backside of the molecule to the active site for hydrolysis reaction. To confirm the water-supplying channel, we blocked the channel by mutating Ala257 at the entrance of the channel to either leucine, phenylalanine or tryptophan. In a subsequent enzyme activity assay, the mutants showed a gradual loss of activity with an increase in size of the hydrophobic side chain (Ala < Leu < Phe < Trp) (Fig. 4B). Thus, our results indicate the presence of a water-supplying channel, which is indispensable for enzymatic function of ACP6.

\section{Substrate recognition and catalysis}

LPA is a series of phospholipids with different lengths and degrees of unsaturation of the acyl chain. Firstly, we studied the enzyme activity of ACP6 on different length of saturated LPAs, plus G3P which lacks the fatty acyl chain of LPA (Fig. 4E). Our results indicated that G3P could not be hydrolyzed by ACP6, suggesting that the fatty acyl chain is indispensable for substrate recognition. Furthermore, our results showed that the activity increased with the length of acyl chain to a peak value on 14:0-LPA, which suggests that a longer acyl chain can help with substrate binding, and that 14:0-LPA is the most favorable substrate in terms of its length. However, the activity gradually decreased again, when the substrate length was increased above the optimal 14:0-LPA, which suggests that these sub- strates are too long to fit into the pocket. Next, the effect of unsaturated bonds was studied by comparing the results of 18:1-LPA and 18:0-LPA (Fig. 4E). Surprisingly, 18:1-LPA exhibited twice the activity of 18:0-LPA. This is because the carboncarbon double bond in 18:1-LPA induces a sharp turn in the overall conformation of LPA, facilitating the accommodation of such a long chain in a favorable conformation in the substratebinding pocket.

To investigate how the substrate binds within the pocket, we introduced obstacles inside the right half of the pocket, by constructing the S285W and L289W mutants (Fig. 5D). The enzyme activity results showed that 6:0-LPA was not affected, 14:0-LPA preserved only half of the wild type activity, yet longer LPAs exhibited almost no activity (Fig. 4E), which suggests that the right half of the pocket participates in binding the acyl chain of substrates longer than 14:0-LPA. Note that the hydrophilic helix $\alpha 1$ at the entrance of the pocket in ACP6 is an additional structural feature, which is absent in other histidine acid phosphatases, and it is flexible in its position. To investigate the function of this specific helix, a multipoint mutant M106112 (YDSQYHE $\rightarrow$ AASAAAA) was constructed and tested (Fig. 4C). We observed that this mutant lost nearly all activity compared to wild type, which confirmed the participation of a1 in substrate recognition. Furthermore, two tyrosine residues Tyr106 and Tyr110 on a1 were analyzed and considered to interact with the substrate through hydroxyl groups, as based on their positions in the structure (Fig. 5D). This prediction was confirmed by weakened activity of mutants carrying either two single mutations Y106F, Y110F, or one double mutation Y(106, 110)F (Fig. 4C).

Several substances such as propranolol, sodium fluoride and $\mathrm{N}$-ethylmaleimide have previously been described as phosphatase inhibitors (Jamal et al., 1991; Kanoh et al., 1992). 
Our inhibition studies showed that addition of propranolol and sodium fluoride resulted in complete inhibition of ACP6 activity, with a 10 times dose of substrate, whilst $\mathrm{N}$-ethylmaleimide inhibited its activity by only about 30\% (Fig. 4D). Moreover, the inhibitory effect of citrate, L-(+)-tartrate and malonate which appeared either in our structures or in the crystallization condition was tested (Fig. 4D). However, no inhibitory effects on enzyme activity were observed. Note that L-(+)-tartaric acid is also considered as an inhibitor for histidine acid phosphatases (Van Etten and Saini, 1978; Ostanin et al., 1994). Nevertheless, it exhibited no inhibitory effects on ACP6, at least not under the neutral $\mathrm{pH}$ conditions tested.

Previously, it was shown that Triton X-100 was necessary for the ACP6 enzyme activity assay; however, its exact role was not examined. As we know, the steps of catalysis include substrate recognition, hydrolysis, $\mathrm{P}_{\mathrm{i}}$ releasing and MAG dissociation. In order to disseminate the specific step of catalysis at which Triton X-100 is involved, we modified the activity assay by increasing the protein amount equal to LPA (from $2 \times 10^{-3}$ $\mathrm{nmol}$ to $50 \mathrm{nmol}$ ) and tested the ACP6 activity without Triton X-100 (Fig. 4F). The results showed a production of $\mathrm{P}_{\mathrm{i}}$ close to $50 \mathrm{nmol}$, revealing that nearly all of the LPA in the reaction system was hydrolyzed without the help of the detergent, which suggests that Triton X-100 does not affect the actual steps of substrate recognition, hydrolysis and $\mathrm{P}_{\mathrm{i}}$ releasing. To confirm the effect of Triton X-100 on the last step, we tested the enzyme activity for different LPAs in the absence of this detergent (Fig. 4E). Of all the different LPAs, only 6:0-LPA exhibited activity. Given that all the products of MAGs are insoluble except for the product of 6:0-LPA, the role of Triton X-100 appears to be at the stage of dissociation and solubilization of the MAGs.

\section{DISCUSSION}

ACP6 was isolated and characterized many years ago ( $\mathrm{Hi}-$ royama and Takenawa, 1998, 1999). However, its substrate recognition and catalytic mechanism have not been studied before. Whilst several homologous structures are available from the database to explain these questions from a general histidine acid phosphatase family perspective, it is important to take into account differences in amino acid composition, resulting in enzyme-specific substrate preferences, and structurefunction particularities. Here, we solved the crystal structure of ACP6, the first structure of a lysophosphatidic acid-specific phosphatase. ACP6 shares several structural elements of significant similarity with other histidine acid phosphatases, among which hPAP is the most closely related. However, hPAP forms a dimeric structure, which is the basis for its catalytic activity, whereas its monomeric form is essentially inactive (Kuciel et al., 1990). Interestingly, the additional fragment 77-116 in ACP6 forms a triangular structure and blocks the corresponding dimer interface. Since ACP6 exists only as a monomer, the question arose as to how activity is established. It was hypothesized that dimerization of PAP could affect the positioning of the interacting helix, which represents the scaffold for the active Arg110 (Jakob et al., 2000). In ACP6, a part of fragment 77-116 (residues 85-94) resembles the interacting fragment in the other dimeric subunit of PAP and covers helix $\alpha 3$ (Fig. 3A and 3B). Through this mechanism, Arg168 is kept in the right position, and thus enabling the enzyme to establish activity even as a monomeric molecule.

By analysis of its signal peptide sequence, ACP6 was confirmed to be localized and to function in the mitochondria. It was shown previously that deletion of its signal peptide caused the loss of activity (Hiroyama and Takenawa, 1999). Intriguingly, the wild type ACP6 exhibits normal activity in our enzyme activity studies, even when the signal peptide was deleted. Though ACP6 is an acid phosphatase, the optimal pH on LPA degradation is 7.5 , and activity is maintained over a wide range, between $\mathrm{pH} 6.0$ and 8.5 (Hiroyama and Takenawa, 1998). In contrast, the catalytic activity of homologous rat PAP and human LAP on $p$-nitrophenyl phosphate (PNPP) is highest at pH 5.4 and 3.0, respectively (Porvari et al., 1994). Note that two polar residues, Tyr123 and Arg127 are located at the entrance of the substrate-binding pocket in rPAP, whereas the corresponding residues in LAP are lysine and glycine (Schneider et al., 1993). The Y123K and R127G double mutant of rPAP shifted the optimal pH to 4.5 (Porvari et al., 1994), which implies that the optimal $\mathrm{pH}$ for catalysis may be related to the distribution of positively charged residues at the entrance. In ACP6, there are three arginines at positions 212, 216 and 219, making up a positively charged area at the entrance of the pocket (Fig. $5 \mathrm{C}$ ), which may be the underlying reason for the observed $\mathrm{pH}$ profile of ACP6.

Our crystal structures clearly indicated that L-(+)-tartrate, malonate and tris formed complexes with ACP6, and bound to its active site. Surprisingly, our results indicated that none of them inhibited the enzyme activity of ACP6 under neutral $\mathrm{pH}$ condition. Previous reports considered that L-(+)-tartaric acid is an inhibitor of histidine acid phosphatase. It also worked for ACP6, however, by changing the $\mathrm{pH}$ value lower to 3.5 which actually caused the loss of activity. Whilst L-(+)-tartrate did not affect the $\mathrm{pH}$ value of the reaction system as well as the enzyme activity. One possible explanation for these results could be that, all the three ligands are able to bind to the active site, yet their binding affinities may not be high enough to inhibit the enzyme activity of ACP6. By measuring the interaction distance between $\mathrm{L}-(+)$-tartrate and surrounding residues, we found that L-(+)-tartrate was bound more loosely in ACP6 than in FtHAP (the average distances are $2.95 \AA$ and $2.89 \AA$, respectively).

To elucidate the molecular details of the interaction between proteins and LPA, several structures of LPA-protein complexes have been solved. For instance, Ex-FABP forms a $\beta$-barrel structure with 14:0-LPA, where it binds in the central channel (Correnti et al., 2011). In the case of anti-LPA antibody (LT3015), dimerization creates the binding channel to accommodate 14:0-LPA, leaving a tail protruding out of the channel (Fleming et al., 2011). In contrast, autotaxin accommodates the body of LPA in a groove, with the tail completely buried in a nearby 
deep hole (Nishimasu et al., 2011). However, dissimilar to the above-described structures of a channel, a hole or even a groove, we have shown that ACP6 accommodates the bent LPAs in a large pocket in various conformations due to the different lengths and degrees of unsaturation of LPA, which was supported by the various enzyme activities on LPAs. A similar relationship has been described between LPA structure and its binding for a series of ATX-LPA complexes (Nishimasu et al., 2011).

The binding modes of the three molecules in our ACP6 structures implied the binding manner of $\mathrm{P}_{\mathrm{i}}$, and a catalytic mechanism similar to that of other histidine acid phosphatases. Three arginines, Arg58, Arg62, Arg168, and one histidine, His334, are positively charged to allow for $\mathrm{P}_{\mathrm{i}}$ binding; His59 is very likely to be the enzyme nucleophile attacking $P_{i}$; and Asp335 may activate a nearby water molecule from the channel, which would then protonate the leaving alcohol group of the substrate, then attack the phosphoenzyme intermediate and finally release $\mathrm{P}_{\mathrm{i}}$. However, to date, no reports are published for histidine acid phosphatases discussing how water is supplied during catalysis. Upon closer inspection of the cutaway view of the ACP6 molecule, an electron density of a water molecule chain was clearly visible inside the hole structure. As expected, this hole was confirmed to be a water-supplying channel by our mutagenesis studies. Therefore, we suggest that holes of similar structure as described for PAP and FtHAP could fulfill similar function. However, some homologous phosphatases that hydrolyze hydrophilic substrates, including phytase and glucose-1-phosphatase, do not possess such structure. Thus, we propose that water is supplied exclusively from their hydrophilic substrate-binding pockets. In contrast, for ACP6, PAP and FtHAP, water could also be supplied from this pocket structure; however, competition with a hydrophobic substrate from the same path does not constitute an effective way for enzyme reaction. Therefore, we propose that the hole opens in a yet unidentified manner that facilitates the supply of water molecules, other than through directly entering the hydrophobic pocket. Moreover, considering the role of Triton $\mathrm{X}-100$, we conclude from our data that there should be an assistant molecule in vivo to help the dissociation and solubilization of the reaction product, MAG.

In conclusion, since LPA is an important signaling molecule involved in many cell activities, and because it also modulates the composition and function of lipid rafts, our structural and primary biochemical studies of ACP6 have provided crucial clues on its substrate recognition and catalytic mechanism, which should contribute to a better understanding of LPA degradation by ACP6. However, the structure of ACP6 complexed with LPA is still needed to uncover the details. On the other hand, mitochondria are known as functionally important organelles, not only for supplying cellular energy, but also for being involved in tasks such as signaling, cellular differentiation, cell death, as well as the control of the cell cycle and cell growth (McBride et al., 2006). Because mitochondria are dynamic organelle constantly undergoing fission and fusion events, they require a constant and well-regulated supply of phospholipids for membrane integrity (Twig et al., 2008; Osman et al., 2011). Moreover, this organelle also plays a role in inter-organelle trafficking of the intermediates and products of phospholipid biosynthetic pathways (Lebiedzinska et al., 2009; Osman et al., 2011). To our knowledge, ACP6 is highly expressed in mitochondria-rich cells, where it regulates the mitochondrial functions by modulating phospholipids of its membrane. However, little else is known about the precise connection between ACP6 activity and mitochondrial functions. Therefore, based on our work and previous studies, more efforts will be needed to comprehensively characterize the role of ACP6 in the mitochondria.

\section{MATERIALS AND METHODS}

Molecular cloning

The DNA sequence encoding human ACP6 without signal peptide (residues 33-428) was amplified from a cDNA clone BC034686 by PCR. The PCR was performed on a thermal cycler (BioRad) with procedure as follows: $95^{\circ} \mathrm{C}$ for $30 \mathrm{~s} ; 55^{\circ} \mathrm{C}$ for $30 \mathrm{~s} ; 72^{\circ} \mathrm{C}$ for $90 \mathrm{~s}$ for 35 cycles, with two primers as listed in Table S1. The product was subcloned into expression vector pGEX-6p-1 (GE Lifesciences), after digestion with $\mathrm{BamHI}$ and $\mathrm{Xhol}$ (TaKaRa). The plasmid containing coding sequence of ACP6 (residues 33-428) was then transformed into E.coli BL21 (DE3) for protein expression.

\section{Site-directed mutagenesis}

Mutants were prepared by PCR with the native ACP6 expression plasmid as a template. Primers were designed for different mutagenesis as listed in Table S1. All mutants were constructed as per TaKaRa MutanBEST Kit instructions. DNA sequencing for constructs were performed to validate successful mutagenesis.

\section{Expression and purification of ACP6}

GST-tagged ACP6 (residues 33-428) protein was expressed at $16^{\circ} \mathrm{C}$, upon induction with $0.5 \mathrm{mmol} / \mathrm{L}$ Isopropyl $\beta$-D-thiogalactoside for $20 \mathrm{~h}$. Cells were harvested after centrifugation at $4600 \mathrm{~g}$ for $30 \mathrm{~min}$, resuspended with $1 \times$ PBS, $\mathrm{pH} 7.4$, lysed by sonication, and centrifuged at $30,000 \mathrm{~g}$ for $30 \mathrm{~min}$ to remove cell debris. The supernatant was collected and passed through a column loaded with Glutathione Sepharose 4B beads. GST-ACP6 was eluted with buffer containing $15 \mathrm{mmol} / \mathrm{L}$ reduced glutathione, $50 \mathrm{mmol} / \mathrm{L}$ Tris- $\mathrm{HCl}, \mathrm{pH} 7.0$, and $150 \mathrm{mmol} / \mathrm{L}$ $\mathrm{NaCl}$. The GST tag was cleaved with PreScission Protease (GE Lifesciences) at $4^{\circ} \mathrm{C}$ overnight, after the protein was dialysed in cleavage buffer $(50 \mathrm{mmol} / \mathrm{L}$ Tris-HCl, pH 7.0, $150 \mathrm{mmol} / \mathrm{L} \mathrm{NaCl}, 1 \mathrm{mmol} / \mathrm{L}$ EDTA, $1 \mathrm{mmol} / \mathrm{L} \mathrm{DTT}$ ), and the tag was removed by passing through Glutathione Sepharose 4B beads once more. ACP6 was exchanged in buffer containing $50 \mathrm{mmol} / \mathrm{L}$ Tris- $\mathrm{HCl}, \mathrm{pH} 8.0$, and $50 \mathrm{mmol} / \mathrm{L} \mathrm{NaCl}$, applied to a column of Hitrap Q HP equilibrated with the same buffer, and eluted with a $0.05-1 \mathrm{~mol} / \mathrm{L}$ linear gradient of $\mathrm{NaCl}$. Fractions were pooled and concentrated and further purified on a Superdex75 10/300GL gel filtration column equilibrated with buffer containing $20 \mathrm{mmol} / \mathrm{L}$ Tris$\mathrm{HCl}, \mathrm{pH} 8.0$, and $150 \mathrm{mmol} / \mathrm{L} \mathrm{NaCl}$. Proteins were collected with high purity and homogeneity and concentrated to $10 \mathrm{mg} / \mathrm{mL}$ for crystalliza- 
tion. SeMet labeled ACP6 was expressed in methionine-free media supplemented with $60 \mathrm{mg} / \mathrm{L} \mathrm{L-SeMet}$, with the purification method being the same as for native protein. Mutant proteins were purified according to the same purification protocol.

\section{Crystallization}

Crystal screening experiments were first carried out at $16^{\circ} \mathrm{C}$ by the mosquito crystallization setting robot (TTP LabTech), with a "hanging drop" vapor diffusion method by mixing $200 \mathrm{~nL}$ of protein solution $(10 \mathrm{mg} / \mathrm{mL}$ in $20 \mathrm{mmol} / \mathrm{L}$ Tris- $\mathrm{HCl}, \mathrm{pH} 8.0$ and $150 \mathrm{mmol} / \mathrm{L} \mathrm{NaCl}$ ) and $200 \mathrm{~nL}$ reservoir solution from commercial crystal screen kits. Crystals appeared the next day in some conditions. Three conditions (Condition 1: $0.2 \mathrm{~mol} / \mathrm{L}$ sodium tartrate dibasic dihydrate, $20 \%(w / v)$ PEG3350; Condition 2: $0.2 \mathrm{~mol} / \mathrm{L}$ sodium malonate, $20 \%$ ( $w / v)$ PEG3350, $\mathrm{pH} 7.0$; Condition 3: $0.02 \mathrm{~mol} / \mathrm{L}$ citric acid, $0.08 \mathrm{~mol} / \mathrm{L}$ Bis-Tris propane, $16 \%$ $(w / v)$ PEG3350, pH 8.8) were optimized, before crystals could grow bigger, and were qualified for X-ray diffraction experiment. Crystals of SeMet-ACP6 were grown under the same conditions.

\section{Data collection and structure determination}

X-ray diffraction data were collected at $100 \mathrm{~K}$ on beamline BL17a at KEK (Tsukuba, Japan). The structure of ACP6 was determined using single-wavelength anomalous diffraction (SAD) method. Two data sets of SeMet-ACP6 (ACP6-malonate and ACP6-tris) were collected at wavelength $0.98 \AA$ and both were processed to $2.20 \AA$ resolution with the HKL2000 software (Otwinowski and Minor, 1997). Ten of the fourteen expected selenium sites were located using the ShelxD program (Schneider and Sheldrick, 2002). Phasing, model building and structure refinement were performed using Phenix software suite (Echols et al., 2012). The models were further refined manually using Coot software (Emsley and Cowtan, 2004). Another native data set (ACP6-L(+)-tartrate) was collected at $1.00 \AA$ wavelength and processed to 2.17 $\AA$ resolution, and the phase was solved by Phaser program in CCP4 program package via molecular replacement (MR) method using the above refined ACP6-tris structure as searching model (Potterton et al., 2003; McCoy et al., 2007). The refinement strategy after MR was the same as the other two models. Data collection and refinement statistics are provided in Table 1. Secondary structures were defined using the DSSP program (Kabsch and Sander, 1983). Structural figures were prepared using Pymol software (Schrodinger, 2010). Structural similarity searches were performed using the Dali server (Holm and Rosenstrom, 2010). Sequence alignment was performed using the program Clustal W (Larkin et al., 2007), and the alignment figures were generated by the ESPript server (Gouet et al., 2003).

\section{Phosphatase activity assay}

The first step of the phosphatase activity assay was the enzyme reaction in a $50 \mu \mathrm{L}$ mixture containing $100 \mathrm{ng}$ enzyme, $50 \mathrm{nmol}$ substrate and $200 \mathrm{nmol}$ Triton X-100 together with buffer containing $20 \mathrm{mmol} / \mathrm{L}$ Tris- $\mathrm{HCl}, \mathrm{pH} 8.0$, and $150 \mathrm{mmol} / \mathrm{L} \mathrm{NaCl}$. Then, the product of $\mathrm{P}_{\mathrm{i}}$ was separated and quantitatively measured as described before (Black and Jones, 1983). In brief, after reaction at $37^{\circ} \mathrm{C}$ for $15 \mathrm{~min}, 200 \mu \mathrm{L}$ chloroform/methanol (1:2) was added, then $80 \mu \mathrm{L}$ chloroform and $80 \mu \mathrm{L} 1 \mathrm{~N} \mathrm{HCl}$ were further added. It was mixed vigorously, and then, after centrifuged at $1200 \mathrm{~g}$ for $5 \mathrm{~min}$, the mixture separated to two phases.
$125 \mu \mathrm{L}$ of the upper phase was transferred into another eppendorf tube. $25 \mu \mathrm{L}$ perchloric acid, $25 \mu \mathrm{L} 10 \%(w / V)$ ammonium molybdate and $50 \mu \mathrm{L} 10 \%(w / V)$ ascorbic acid were added and then the mixture was boiled at $95^{\circ} \mathrm{C}$ for $5 \mathrm{~min}$. The absorbance was measured at $795 \mathrm{~nm}$ with a VarioshanFlash spectrophotometer (Thermo). Inhibition assays were carried out by adding $0.5 \mu \mathrm{mol}$ potential inhibitor into the enzyme reaction system before enzyme activity detection.

\section{ACCESSION NUMBERS}

The coordinates and structure factors have been deposited in the PDB database. Accession codes: 4JOB, 4JOC and 4JOD.

\section{ACKNOWLEDGEMENTS}

We are grateful to Dr. Xuemei Li and Dr. Yutao Chen for their helpful discussion. We would like to thank staff members of Core Facility for Protein Research in the Institute of Biophysics, CAS, especially Yi Han, Ya Wang and Xiaoxia Yu for their technical support. This work was supported by grants from the State Key Development Program for Basic Research of the Ministry of Science and Technology of China (973 Project) (Grant Nos. 2011CB915501 and 2011CB910304) and National Infectious disease Funding (Grant No. 2012ZX10004701).

\section{ABBREVIATIONS}

3'-AMP, adenosine 3'-phosphate; 5'-AMP, adenosine 5'-phosphate; ACP6, lysophosphatidic acid phosphatase type 6; ATX, autotoxin; DTT, dithiothreitol; EDTA, ethylene diamine tetraacetic acid; Ex-FABP, extracellular fatty acid binding protein; FtHAP, Francisella tularensis histidine acid phosphatase; G3P, glycerol-3-phosphate; GST, glutathione Stransferase; LAP, lysosomal acid phosphatase; LPA, lysophosphatidic acid; LPC, lysophosphatidylcholine; MAG, monoacylglycerol; MR, molecular replacement; NEM, N-ethylmaleimide; $\mathrm{P}_{\mathrm{i}}$, inorganic phosphate; PA, phosphatidic acid; PAP, prostatic acid phosphatase; PDB, Protein Data Bank; PEG, polyethylene glycol; PNPP, $p$-nitrophenyl phosphate; r.m.s.d., root mean square deviation; SAD, single-wavelength anomalous diffraction; SeMet, selenomethionine

\section{COMPLIANCE WITH ETHICS GUIDELINES}

Jun Li, Yu Dong, Xingru Lü, Lu Wang, Wei Peng, Xuejun C. Zhang, and Zihe Rao declare that they have no conflict of interest.

This article does not contain any studies with human or animal subjects performed by the any of the authors.

\section{REFERENCES}

Ando, T., Ishiguro, H., Kuwabara, Y., Kimura, M., Mitsui, A., Kurehara, H., Sugito, N., Tomoda, K., Mori, R., Takashima, N., et al. (2006). Expression of ACP6 is an independent prognostic factor for poor survival in patients with esophageal squamous cell carcinoma. Oncol Rep 15, 1551-1555.

Athenstaedt, K., Weys, S., Paltauf, F., and Daum, G. (1999). Redundant systems of phosphatidic acid biosynthesis via acylation of glycerol-3-phosphate or dihydroxyacetone phosphate in the yeast Saccharomyces cerevisiae. J Bacteriol 181, 1458-1463.

Bell, R.M., and Coleman, R.A. (1980). Enzymes of glycerolipid synthesis in eukaryotes. Annu Rev Biochem 49, 459-487. 
Black, M.J., and Jones, M.E. (1983). Inorganicphosphate determination in the presence of a labile organic phosphate: assay for carbamylphosphate phosphataseactivity. Anal Biochem 135, 233-238.

Blackburn, J., and Mansell, J.P. (2012). The emerging role of lysophosphatidic acid (LPA) in skeletal biology. Bone 50, 756-762.

Correnti, C., Clifton, M.C., Abergel, R.J., Allred, B., Hoette, T.M., Ruiz, M., Cancedda, R., Raymond, K.N., Descalzi, F., and Strong, R.K. (2011). Galline Ex-FABP is an antibacterial siderocalin and a lysophosphatidic acid sensor functioning through dual ligand specificities. Structure 19, 1796-1806.

Echols, N., Grosse-Kunstleve, R.W., Afonine, P.V., Bunkoczi, G., Chen, V.B., Headd, J.J., McCoy, A.J., Moriarty, N.W., Read, R.J., Richardson, D.C., et al. (2012). Graphical tools for macromolecular crystallography in PHENIX. J Appl Crystallogr 45, 581-586.

Emsley, P., and Cowtan, K. (2004). Coot: model-building tools for molecular graphics. Acta Crystallogr D Biol Crystallogr 60, 2126-2132.

Fleming, J.K., Wojciak, J.M., Campbell, M.A., and Huxford, T. (2011). Biochemical and structural characterization of lysophosphatidic Acid binding by a humanized monoclonal antibody. J Mol Biol 408, 462-476.

Gaits, F., Fourcade, O., Le Balle, F., Gueguen, G., Gaige, B., Gassama-Diagne, A., Fauvel, J., Salles, J.P., Mauco, G., Simon, M.F., et al. (1997). Lysophosphatidic acid as a phospholipid mediator: pathways of synthesis. FEBS Lett 410, 54-58.

Gouet, P., Robert, X., and Courcelle, E. (2003). ESPript/ENDscript: Extracting and rendering sequence and $3 \mathrm{D}$ information from atomic structures of proteins. Nucleic Acids Res 31, 3320-3323.

Hiroyama, M., and Takenawa, T. (1998). Purification and characterization of a lysophosphatidic acid-specific phosphatase. Biochem J 336, 483-489.

Hiroyama, M., and Takenawa, T. (1999). Isolation of a cDNA encoding human lysophosphatidic acid phosphatase that is involved in the regulation of mitochondrial lipid biosynthesis. J Biol Chem 274, 29172-29180.

Holm, L., and Rosenstrom, P. (2010). Dali server: conservation mapping in 3D. Nucleic Acids Res 38, W545-549.

Jakob, C.G., Lewinski, K., Kuciel, R., Ostrowski, W., and Lebioda, L. (2000). Crystal structure of human prostatic acid phosphatase. Prostate 42, 211-218.

Jamal, Z., Martin, A., Gomez-Munoz, A., and Brindley, D.N. (1991). Plasma membrane fractions from rat liver contain a phosphatidate phosphohydrolase distinct from that in the endoplasmic reticulum and cytosol. J Biol Chem 266, 2988-2996.

Kabsch, W., and Sander, C. (1983). Dictionary of protein secondary structure: pattern recognition of hydrogen-bonded and geometrical features. Biopolymers 22, 2577-2637.

Kai, M., Wada, I., Imai, S., Sakane, F., and Kanoh, H. (1996). Identification and cDNA cloning of 35-kDa phosphatidic acid phosphatase (type 2) bound to plasma membranes. Polymerase chain reaction amplification of mouse $\mathrm{H}_{2} \mathrm{O}_{2}$-inducible hic53 clone yielded the cDNA encoding phosphatidic acid phosphatase. J Biol Chem 271, 18931-18938.

Kanoh, H., Imai, S., Yamada, K., and Sakane, F. (1992). Purification and properties of phosphatidic acid phosphatase from porcine thymus membranes. J Biol Chem 267, 25309-25314.

Kanoh, H., Iwata, T., Ono, T., and Suzuki, T. (1986). Immunological characterization of sn-1,2-diacylglycerol and sn-2-monoacylglycerol kinase from pig brain. J Biol Chem 261, 5597-5602.

Krissinel, E., and Henrick, K. (2007). Inference of macromolecular assemblies from crystalline state. J Mol Biol 372, 774-797.

Kuciel, R., Bakalova, A., Mazurkiewicz, A., Bilska, A., and Ostrowski, W. (1990). Is the subunit of prostatic phosphatase active? Reversible denaturation of prostatic acid phosphatase. Biochem Int 22, 329-334.

LaCount, M.W., Handy, G., and Lebioda, L. (1998). Structural origins of $\mathrm{L}(+)$-tartrate inhibition of human prostatic acid phosphatase. J Biol Chem 273, 30406-30409.

Larkin, M.A., Blackshields, G., Brown, N.P., Chenna, R., McGettigan, P.A., McWilliam, H., Valentin, F., Wallace, I.M., Wilm, A., Lopez, R., et al. (2007). Clustal $W$ and Clustal $X$ version 2.0. Bioinformatics 23, 2947-2948.

Lebiedzinska, M., Szabadkai, G., Jones, A.W., Duszynski, J., and Wieckowski, M.R. (2009). Interactions between the endoplasmic reticulum, mitochondria, plasma membrane and other subcellular organelles. Int J Biochem Cell Biol 41, 1805-1816.

Lee, D.C., Cottrill, M.A., Forsberg, C.W., and Jia, Z. (2003). Functional insights revealed by the crystal structures of Escherichia coli glucose-1-phosphatase. J Biol Chem 278, 31412-31418.

Lim, D., Golovan, S., Forsberg, C.W., and Jia, Z. (2000). Crystal structures of Escherichia coli phytase and its complex with phytate. Nat Struct Biol 7, 108-113.

Matthews, B.W. (1968). Solvent content of protein crystals. J Mol Biol 33, 491-497.

McBride, H.M., Neuspiel, M., and Wasiak, S. (2006). Mitochondria: more than just a powerhouse. Curr Biol 16, R551-560.

McCoy, A.J., Grosse-Kunstleve, R.W., Adams, P.D., Winn, M.D., Storoni, L.C., and Read, R.J. (2007). Phaser crystallographic software. J Appl Crystallogr 40, 658-674.

Mills, G.B., and Moolenaar, W.H. (2003). The emerging role of lysophosphatidic acid in cancer. Nat Rev Cancer 3, 582-591.

Moolenaar, W.H. (1994). LPA: a novel lipid mediator with diverse biological actions. Trends Cell Biol 4, 213-219.

Moolenaar, W.H., Jalink, K., and van Corven, E.J. (1992). Lysophosphatidic acid: a bioactive phospholipid with growth factor-like properties. Rev Physiol Biochem Pharmacol 119, 47-65.

Nishimasu, H., Okudaira, S., Hama, K., Mihara, E., Dohmae, N., Inoue, A., Ishitani, R., Takagi, J., Aoki, J., and Nureki, O. (2011). Crystal structure of autotaxin and insight into GPCR activation by lipid mediators. Nat Struct Mol Biol 18, 205-212.

Ortlund, E., LaCount, M.W., and Lebioda, L. (2003). Crystal structures of human prostatic acid phosphatase in complex with a phosphate ion and alpha-benzylaminobenzylphosphonic acid update the mechanistic picture and offer new insights into inhibitor design. Biochemistry 42, 383-389.

Osman, C., Voelker, D.R., and Langer, T. (2011). Making heads or tails of phospholipids in mitochondria. J Cell Biol 192, 7-16.

Ostanin, K., Saeed, A., and Van Etten, R.L. (1994). Heterologous expression of human prostatic acid phosphatase and site-directed mutagenesis of the enzyme active site. J Biol Chem 269, 89718978.

Otwinowski, Z., and Minor, W. (1997). Processing of X-ray diffraction data collected in oscillation mode. In Elsevier, pp. 307-326.

Porvari, K.S., Herrala, A.M., Kurkela, R.M., Taavitsainen, P.A., Lindqvist, Y., Schneider, G., and Vihko, P.T. (1994). Site-directed 
mutagenesis of prostatic acid phosphatase. Catalytically important aspartic acid 258, substrate specificity, and oligomerization. J Biol Chem 269, 22642-22646.

Potterton, E., Briggs, P., Turkenburg, M., and Dodson, E. (2003). A graphical user interface to the CCP4 program suite. Acta Crystallogr D Biol Crystallogr 59, 1131-1137.

Reddy, V.S., Rao, D.K., and Rajasekharan, R. (2010). Functional characterization of lysophosphatidic acid phosphatase from Arabidopsis thaliana. Biochim Biophys Acta 1801, 455-461.

Schneider, G., Lindqvist, Y., and Vihko, P. (1993). Three-dimensional structure of rat acid phosphatase. EMBO J 12, 2609-2615.

Schneider, T.R., and Sheldrick, G.M. (2002). Substructure solution with SHELXD. Acta Crystallogr D Biol Crystallogr 58, 1772-1779.

Schrodinger, LLC (2010). The PyMOL Molecular Graphics System, Version 1.3r1.

Shekar, S., Tumaney, A.W., Rao, T.J., and Rajasekharan, R. (2002). Isolation of lysophosphatidic acid phosphatase from developing peanut cotyledons. Plant Physiol 128, 988-996.

Singh, H., Felts, R.L., Schuermann, J.P., Reilly, T.J., and Tanner, J.J. (2009). Crystal Structures of the histidine acid phosphatase from Francisella tularensis provide insight into substrate recognition. $J$ Mol Biol 394, 893-904.

Thompson, F.J., and Clark, M.A. (1994). Purification of a lysophosphatidic acid-hydrolysing lysophospholipase from rat brain. Biochem $\mathrm{J}$ 300, 457-461.

Thomson, F.J., and Clark, M.A. (1995). Purification of a phosphatidicacid-hydrolysing phospholipase A2 from rat brain. Biochem J 306, 305-309.

Tokumura, A., Majima, E., Kariya, Y., Tominaga, K., Kogure, K., Yas- uda, K., and Fukuzawa, K. (2002). Identification of human plasma lysophospholipase $D$, a lysophosphatidic acid-producing enzyme, as autotaxin, a multifunctional phosphodiesterase. J Biol Chem 277, 39436-39442.

Twig, G., Elorza, A., Molina, A.J., Mohamed, H., Wikstrom, J.D., Walzer, G., Stiles, L., Haigh, S.E., Katz, S., Las, G., et al. (2008). Fission and selective fusion govern mitochondrial segregation and elimination by autophagy. EMBO Journal 27, 433-446.

Umezu-Goto, M., Kishi, Y., Taira, A., Hama, K., Dohmae, N., Takio, K., Yamori, T., Mills, G.B., Inoue, K., Aoki, J., et al. (2002). Autotaxin has lysophospholipase $\mathrm{D}$ activity leading to tumor cell growth and motility by lysophosphatidic acid production. J Cell Biol 158, 227-233.

Van Etten, R.L., and Saini, M.S. (1978). Selective purification of tartrate-inhibitable acid phosphatases: rapid and efficient purification (to homogeneity) of human and canine prostatic acid phosphatases. Clin Chem 24, 1525-1530.

Waggoner, D.W., Gomez-Munoz, A., Dewald, J., and Brindley, D.N. (1996). Phosphatidate phosphohydrolase catalyzes the hydrolysis of ceramide 1-phosphate, lysophosphatidate, and sphingosine 1-phosphate. J Biol Chem 271, 16506-16509.

Waggoner, D.W., Martin, A., Dewald, J., Gomez-Munoz, A., and Brindley, D.N. (1995). Purification and characterization of novel plasma membrane phosphatidate phosphohydrolase from rat liver. J Biol Chem 270, 19422-19429.

Webber, K.O., and Hajra, A.K. (1992). Dihydroxyacetone phosphate acyltransferase. Methods Enzymol 209, 92-98.

Xie, M., and Low, M.G. (1994). Identification and characterization of an ecto-(lyso)phosphatidic acid phosphatase in PAM212 keratinocytes. Arch Biochem Biophys 312, 254-259. 